\title{
- Dual Energy Computed Tomography in Gout: Our Experience
}

IJCRR
ection: Healthcare
ISI Impact Factor
$(2019-20): 1.628$
Value (2019): 90.81
SJIF (2020) = 7.893
Copyright@IJCRR

\section{Sagdeo A. ${ }^{1}$, Askari A. ${ }^{2}$, Singh J. ${ }^{3}$, Tins B. ${ }^{3}$, Ball P.A. ${ }^{4}$, Morrissey H. ${ }^{5}$}

'Clinical Fellow in Rheumatology, The Robert Jones and Agnes Hunt Orthopaedic Hospital, NHS Foundation Trust, SY10 7 AG United Kingdom; ${ }^{2}$ Consultant Rheumatologist, The Robert]ones and Agnes Hunt Orthopaedic Hospital, NHS Foundation Trust, SY10 7 AG United Kingdom; ${ }^{3}$ Consultant Radiologist, The Robert Jones and Agnes Hunt Orthopaedic Hospital, NHS Foundation Trust, SY10 7 AG United Kingdom; 5Professor of Pharmacy Practice, The University of Wolverhampton, Faculty of Science and Engineering, School of Pharmacy, Wulfruna Street, WV1 $1 \mathrm{LY}$ United Kingdom; ${ }^{6}$ Reader in Clinical pharmacy, The University of Wolverhampton, Faculty of Science and Engineering, School of Pharmacy, Wulfruna Street, WV ı lY United Kingdom.

\section{ABSTRACT}

Introduction: Gout is a common medical problem, affecting at least $1 \%$ of men in Western countries, with a male: female ratio ranging from 7:1 to 9:1. For many patients, traditional investigations can be inconclusive. Dual Energy Computed Tomography (DECT) is emerging as a valuable tool for non-invasive confirmation of urate deposits in painful joints.

Aim: To establish the effectiveness of the DECT in the identification of gout in patients with complex presentations where the diagnosis is not clear.

Method: DECT at $140 \mathrm{kV}$ and $80 \mathrm{kV}$ was used to image patients where the clinical diagnosis was unclear

Results: Seven case studies are presented where an unclear clinical presentation was successfully diagnosed with the use of DECT.

Conclusion: In a specialist tertiary referral centre, treating many patients whose presentation is atypical, DECT has become a valuable tool in confirming the presence or absence of gouty arthritis, in difficult cases with a diagnostic dilemma.

Key Words: Imaging, Dual Energy, X-Ray, Computed Tomography, Gout, Gouty Arthritis, Hyperuricaemia, Rheumatology

\section{INTRODUCTION}

In Western countries, Gout is a common condition reported to affect around $1 \%$ of men with a reported male to female ratio between 7 and 9:1. ${ }^{1}$ The typical presentation is with intermittent, recurrent of mono- or oligoarticular inflammation characterised by very severe pain, however, a much less specific presentation with polyarticular chronic joint pain may occur, complicating the differential diagnosis. ${ }^{1}$

Dual Energy Computed Tomography (DECT) can identify specific elements and molecules such as iodine, calcium and uric acid, providing valuable new diagnostic information. ${ }^{2}$ Imaging and identification of urate deposits provides a novel, rapid, non-invasive and reliable method for the confirmation of gout. ${ }^{3}$

In patients with or without a history of gout and a recent suspicion for gouty arthritis, DECT has a marked diagnostic impact when hyperdense soft-tissue deposits are present. ${ }^{4} \mathrm{~A}$ definitive diagnosis requires the direct identification of urate crystals in the joint and the exclusion of infection. Serum urate levels are frequently normal during attacks of acute gout, so reliance on urate level during acute attacks of gouty arthritis is not useful. ${ }^{5}$ The classic plain radiographical signs such as well defined, punched out, periarticular erosions with overhanging edges, may not be seen until six or even more years after initial attacks. MRI can be very useful, but is expensive and, like conventional computed tomography, lacks specificity. ${ }^{6}$

Ultrasonography can be useful if the classic features of gout are present, being low cost, no radiation exposure, easy availability, and the ability to combine it with aspiration. However, it requires an experienced sonographer and highresolution equipment. ${ }^{7}$ Since none of these imaging modalities can specifically identify the chemical composition of uric acid, a specific display algorithm based on the chemical composition of uric acid by dual-energy CT (DECT) scans is useful as an accurate imaging tool for gout. ${ }^{8}$

\section{Corresponding Author:}

Morrissey H., Reader in Clinical Pharmacy, University of Wolverhampton, Wolverhampton WV1 1LY, United Kingdom. Email: hana.morrissey@wlv.ac.uk, ORCID: https://orcid.org/0000-0001-9752-537X.

ISSN: 2231-2196 (Print)

Received: 04.10 .2020
ISSN: 0975-5241 (Online)

Revised: 24.12 .2020
Accepted: 12.03 .2021
Published: 20.07 .2021 
The colour-coded information of the urate deposits in DECT scans allows for a computer-automated procedure to measure the volume of each clinical or subclinical tophus, which can then be summed to produce a total volume of urate deposition in peripheral joints of a given patient. Thus, DECT scans also carry several potential advantages as a follow-up imaging tool over MRI, which was recently evaluated to quantify tophus size. These advantages include a lower cost $(\sim 1 / 6)$, a shorter scan time ( $\sim 15 \mathrm{~min}$ for all peripheral joints), simultaneous scanning of multiple joints, obvious colour-differential display of crystals and minimal influence of positioning. Furthermore, the MRI volume measurement of tophus size involves the manual tracing of consecutive MR images. ${ }^{6}$

The potential applications of DECT also include establishing the extent of gout by identifying subclinical intra- and extra-articular tophi, evaluation of nodular lesions, diagnosing the concurrent presence of gout in patients with other arthropathies, identifying urate deposits in challenging and atypical anatomical sites of gout, understanding the anatomical distribution of the disease and monitoring the response to treatment through measurement of individual tophus volume and total tophus burden. Of note, OMERACT has proposed tophus regression as a core domain for outcome measures in chronic gout. $^{9}$

The obvious colour display and three-dimensional volumerendered imaging of the disease allow information to be easily and communicated to clinicians and patients.

The radiation exposure of DECT for peripheral joint imaging is minimal. It is estimated that the DECT radiation dose is $0.5 \mathrm{mSv}$ of dose per scanned region and the total amount for all peripheral joints per patient ranges from 2 to $3 \mathrm{mSv}$, which is similar to the annual global per caput average dose due to natural radiation sources $(2.4 \mathrm{mSv}) .{ }^{10}$

Furthermore, the target image areas for gout are radio-insensitive peripheral joints, and the vast majority of patients with gout are predominately middle-aged or elderly men, which supports a minimal risk of radiation associated with this potential modality for gout at a practical level. ${ }^{10}$

DECT scans are possible in patients with severely impaired renal function. Usually, the two scan-energies are set to $140 \mathrm{kV}$ and $80 \mathrm{kV}$. Based on these two imaging series, different crystals with different absorption characteristics can be distinguished using several commercially available software modalities. These software tools allow for colour coded discrimination of urate crystal deposits as well as several other crystal types. DECT can serve as a problem-solving tool in the presence of challenging clinical presentations. ${ }^{11}$

\section{Aim}

To establish the effectiveness of the Dual Energy Computed Tomography in the identification of gout in patients with complex presentations where the diagnosis is not clear.

\section{Method}

This is a gout diagnosis quality improvement audit. This report is based on the review of seven patients records where DECT at $140 \mathrm{kV}$ and $80 \mathrm{kV}$ was used to image patients where the clinical diagnosis was unclear. All possible patient identifiers were removed, with only gender and age was used. Patients verbal approvals were taken to use their pictures in this report.

\section{RESULT AND DISCUSSION}

Case report-1: A 74-year-old male, known case of chronic gout on Allopurinol. His co-morbidities were atrial fibrillation, hypertension and osteoarthritis. This patient presented with increasing pain/swelling in hands particularly the PIPs of both middle fingers that were slightly tender with nodular swellings over PIP and DIP. Uric acid was normal. The patient was anxious regarding his management and the presence of these nodular swellings. He thought that a change of management or increase in the Allopurinol dose is required. It was felt that his pain was due to a degenerative process rather than gout. CT dual-energy confirmed the complete absence of any urate crystals in the hands. We concluded that his chronic pain is caused mainly by the degenerative process. Management focused on physical therapy and the continuation of the same medications.

Case Report 2: A 54-year-old female was referred by her general practitioner with a history of Left foot pain on/off with swelling and redness. Her father and brother both have gout and she was very anxious that her symptoms are due to gout. On investigations her autoimmune screen including anti-CCP and ACE is negative, uric acid was normal. X-rays were unremarkable. DECT showed no evidence of any crystal deposition providing reassurance to the patient. She was advised physical therapy and occasional NSAID.

Case report 3: A 47-year-old female, known case of Fibromyalgia, irritable bowel syndrome and gall stones. She was referred for painful toes in addition to fatigue due to fibromyalgia by her doctor. Examination revealed second toe classic dactylitis with tenderness but no other features of Spondylo-arthropathy. Her blood showed, ESR: $25 \mathrm{~mm} /$ Hr, CRP: $2 \mathrm{mg} / \mathrm{L}$, Uric Acid $512 \mu \mathrm{mol} / \mathrm{L}$. DECT revealed urate crystals in the second MTPJ. No crystals seen around $1^{\text {st }}$ MTPJ and no erosive changes. She was advised to lose weight and pharmacological therapy was considered.

Case report 4: A64-year-old male, known case of psoriatic arthritis and osteoarthritis, complained of severe hand and knee pains interfering with his job for the past 6 months. Clinically, he had mild to moderate tenderness in relevant joints, and could not make a full fist. X-ray showed prominent degenerative in knees and hands but no erosions. Ultrasound (US) showed MCPJ synovitis but no clear underlying 
aetiology. DECT confirmed the presence of uric acid crystals within flexor hand and proximal tendons corresponding clinically with areas of tenderness. Pharmacological treatment was commenced, and he achieved considerable relief.

Case report 5: A 50-year-old male, drinking 35 units of alcohol weekly, was admitted to Hospital for left ankle pain and swelling. No crystals or infection in the synovial fluid. Uric acid level was $474 \mu \mathrm{molL}^{-1}$ and autoimmune tests were negative. Examination revealed second MTPJ swelling but no findings in the ankle or big toes.

DECT confirmed urate crystals in the second toe, Achilles tendon insertion; peroneal tendons and tibiofibular syndesmosis established the diagnosis of gout (Figure 1).

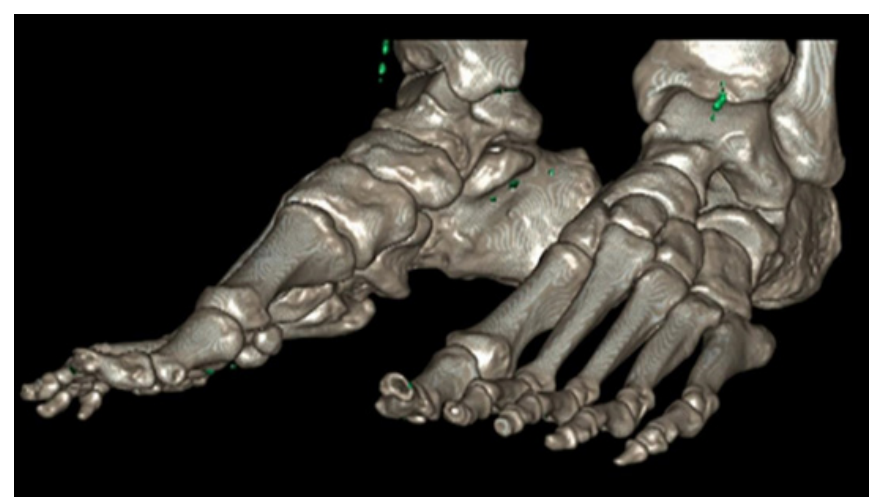

Figure 1: DECT image showing green coloured uric acid deposits in the second toe, Achilles tendon insertion, peroneal tendons and tibio-fabular syndesmosis

Case report 6: A 72-year-old female patient presented with sudden swelling of MCPJs and proximal phalanges in all digits except the thumbs. Examination revealed, mild redness, no other signs of inflammation. The patient was extremely concerned, as she had a probable previous diagnosis of gout. Her uric acid level was highly elevated at $586 \mu \mathrm{molL}^{-1}$, autoimmune screen was negative. US reported fatty tissue with an unusual pattern of deposition. DECT confirmed the unusual fatty deposition and distribution, ruled out tophi, tumours or nodules. Patient was reassured that the swellings were not gout related and the latter should be treated independently.

Case report 7: A 74-year-old male, known case of gout on Allopurinol $300 \mathrm{mg}$, continued drinking alcohol around 60 units weekly. He presented with moderate feet pain in both feet for $>12$ months. On examination he has bilateral MTPJ swelling and tenderness. His ESR was $8 \mathrm{~mm} / \mathrm{Hr}$, CRP was $26 \mathrm{mg} / \mathrm{L}$, autoimmune screen including Anti-CCP is negative and uric acid is normal at $218 \mu \mathrm{mol} / \mathrm{L}$. Intermittent oral steroids helped his symptoms. DECT showed the presence of urate crystals around the MTPJs, mid and hindfoot and ankle tendons confirming the diagnosis of gout (Figure 2).

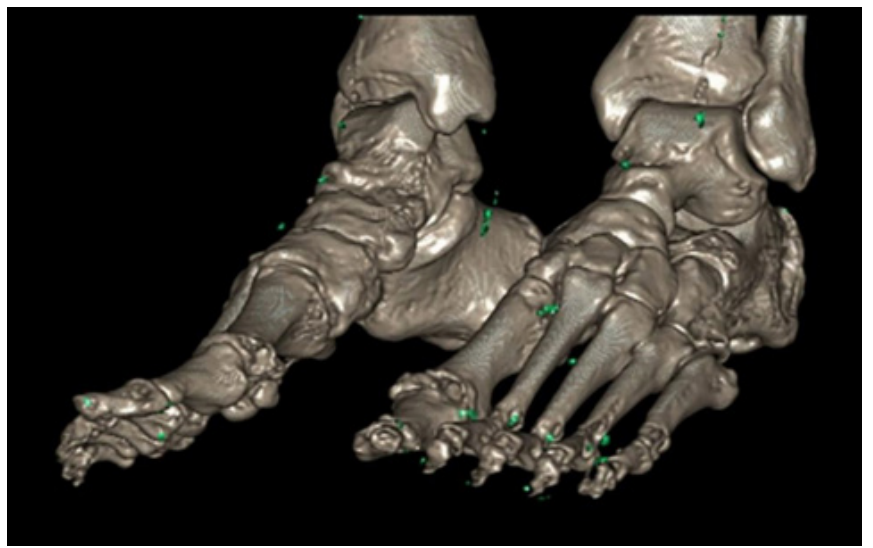

Figure 2: DECT image showing the presence of urate crystals seen as green spots around MTPJ's, midfoot, hindfoot and ankle tendons.

\section{CONCLUSION}

In straightforward cases where there is a typical clinical picture of acute arthritis where septic arthritis is the main differential diagnosis against crystal arthropathy, joint aspiration showing the presence of negatively birefringent monosodium urate crystals, gout would be straightforward to confirm and manage. However, in difficult situations like excessive swelling of peripheral soft tissue and the absence of accessible fluid or tophus, there is a need for a specific and sensitive imaging tool. All our cases posed a diagnostic dilemma that needed confirmation, DECT was very useful to confirm or refute gouty arthritis. In our small case series, DECT, helped us to exclude the diagnosis of gout in cases 1,2 and 6 , whereas in the remaining cases, gout was confirmed.

When the clinical presentation is unclear, DECT is a noninvasive, valuable, underutilised investigation that can establish the diagnosis of gout particularly when other arthritic conditions are present moreover it may rule out gout as a cause of the inflammatory process.

\section{ACKNOWLEDGEMENT}

The authors acknowledge the help received from the scholars whose articles are cited and included in references to this manuscript. The authors are also grateful to authors/ editors/publishers of all those articles, journals and books from where the literature for this article has been reviewed and discussed

Conflict of interest: No conflict of interest known

Funding: No external funding

\section{Authors' contributions:}

Sagdeo A, Askari A, Singh J, Tins B. conducted data collation and clinical interpretation and Ball PA. Morrissey H. 
reviewed the report and drafted the manuscript and author 6 completed the submission.

\section{REFERENCES}

1. Luk AJ, Simkin PA. Epidemiology of hyperuricemia and gout. Am J Manag Care 2005;11(15 Suppl): S435-68.

2. Courtney CA, Nelson RC, Boll DT, Paulson EK, Ho LM, Neville AM, et al. Dual-Energy Multidetector CT: How Does It Work, What Can It Tell Us, and When Can We Use It in Abdominopelvic Imaging? Radiogra. 2010:3(4):1037-52.

3. Bongartz T, Glazebrook KN, Kavros SJ, Murthy NS, Merry SP, Franz WB, et al. Dual-energy CT for the diagnosis of gout: an accuracy and diagnostic yield study. Ann Rheum Dis. 2015;74:1072-7.

4. Finkenstaedt T, Manoliou A, Toniolo M, Higashigaito K, Andreisek G, Guggengerger R, et al. Gouty arthritis: the diagnostic and therapeutic impact of dual-energy CT. Eur Radiol. 2016;26(11):3989-39.
5. Terkeltaub RA, Gout. New Engl J Med Boston. 2003;349(17):1647-55.

6. Schumacher HR Jr, Becker MA, Edwards NL, Palmer, WE, MacDonald PA, Palo W, et al. Magnetic resonance imaging in the quantitative assessment of gouty tophi. Int J Clin Pract. 2006; 60:408-414.

7. Thiele RG, Schlesinger N. Diagnosis of gout by ultrasound. Rheumatic. 2007; 46:1116-21.

8. Johnson TR, Weckbach S, Kellner H, Reiser MF, Becker CR Clinical image: dual-energy computed tomographic molecular imaging of gout. Arthritis Rheum. 2007;56:2809.

9. Schumacher HR Jr, Edwards LN, Perez-Ruiz F, Becker M, Chen LX, Furst DE, et al. Outcome measures for acute and chronic gout. J Rheumatol 2005; 32:2452-5.

10. Choi HK, Al-Arfaj AM, Eftekhari A, Munk PL, Shojania K, Reid G et al. Dual-Energy Computed Tomography in Tophaceous Gout, Ann Rheum Dis. 2009;68(10):1609-12.

11. Nicolaou S, Yong-Hing CJ, Galea-Soler S, Hou DJ, Luck L, Munk P. Dual-energy CT as a potential new diagnostic tool in the management of gout in the acute setting. Am J Roentgenol. 2010;194:1072-8. 